\title{
Image Resolution Enhancement using Multi-wavelet Transforms with Interpolation Technique.
}

\author{
Prof. Mrs. Meenakshi M. Pawar ${ }^{1}$ Miss. Neeta P. Kulkarni ${ }^{2}$ \\ 1, 2 (Dept. Of Electronics \&Tele-communication) \\ SVERI's College of Engineering, Pandharpur / Solapur University ,India
}

\begin{abstract}
Images with high resolution give better results for image processing applications. Image resolution enhancement is the process of manipulating an image so that resultant image is more suitable for specific application such as medical, agricultural, satellite image processing. This paper is based on image resolution enhancement by combination of SWT \& DWT.In DWT main loss is on high frequency components. The edge loss in high frequency components of DWT is minimized by adding it with high frequency components of SWT which is transition invariant transform. The interpolated high frequency sub-bands of DWT are added with high frequency sub bands of SWT. Then the added high frequency sub-bands (of SWT \& DWT) as well as the input image are interpolated by factor of $\alpha / 2$ to enlarge the input image by factor of $\alpha$. Afterwards all these images have been combined using IDWT to generate a super resolved image. Algorithm considering Haar function is developed for DWT \& inverse IDWT instead of using db9/7. This obtained image is compared with original high resolution image. This technique has been tested on standard bench mark images. The quantitative (Peak signal to noise ratio) and visual results are superior over conventional image resolution enhancement techniques.
\end{abstract}

Keywords: Bi-cubic interpolation, discrete wavelet transform, image resolution enhancement, stationary wavelet transform

\section{Introduction}

Image resolution enhancement plays key role in digital image processing so we focus on the importance of the representation of information for various image processing tasks. Resolution of an image is its clear perspective to the front person or machine depending upon its application. In today's world image processing is applied almost in every field of Science. Major of them are medical, agricultural, satellite image processing, video resolution enhancement, RADAR imaging system. The resolution of image is important aspect which will decide performance of application. High resolution image produces good results. An image is represented by intensity at a particular point whose spatial coordinates are on generally $\mathrm{x}$ and $\mathrm{y}$ axis coordinates. The $2 \mathrm{D}$ continuous image is divided into $\mathrm{N}$ rows and $\mathrm{M}$ columns. The intersection of a row and a column is called a pixel.

The resolution or dimension is often used for a pixel count in digital imaging. Interpolation is one of the commonly used technique for image resolution enhancement. It has been widely used in many applications in image processing such as facial reconstruction, multiple description coding, and super resolution. Interpolation is the process of using known data values to estimate unknown data values.

There are three well known interpolation techniques, namely nearest neighbor interpolation, bilinear interpolation, and bi-cubic interpolation. The main disadvantage in using interpolation is the loss of $\mathrm{HF}$ components (Edges), which is due to the smoothing caused by interpolation. Preserving the edges is essential. To avoid this problem we use a new mathematical tool called wavelet transform.

DWT and SWT are the recent wavelet transforms in image processing. Discrete Wavelet Transform (DWT), Stationary wavelet transform (SWT) transform are main versatile tools for modern image processing.

\section{Motivation}

Resolution of an image has been always an important issue in many image and videoprocessing applications, such as video resolution enhancement, feature extraction, and satellite image resolution enhancement. Interpolation in image processing is a method to increase or decrease the number of pixels in a digital image. In this known data values are used to predict unknown data values. Interpolation lags in edge preservation. Also in interpolation as interpolation factor increases the computation also increases. So the alternative approach is to use the image in some other domain. The domain can be Fourier domain, wavelet domain . Fourier domain is more suitable for spectral filtering. But it creates problem when the points are apart. Discrete Cosine transform was next transform but can't be used for lossless image compression. So all these problems are overcome by operating images in wavelet domain. 
Discrete wavelet transform (DWT) is one of the latest [11] wavelet transform. It is simple mathematical tool for signal processing. DWT composes filter bank (High pass filter\& low pass filter).DWT decomposes image into four sub -bands. Image resolution enhancement in the wavelet domain is a relatively new research topic and recently many new algorithms have been proposed [2]-[5]. Another recent wavelet transform which has been used in several image processing applications is stationary wavelet transform (SWT) .In short, SWT is similar to DWT but it does not use down-sampling, hence the sub-bands will have the same size as the input image. The SWT is an inherently redundant scheme as the output of each level of SWT contains the same number of samples as the input. So for a decomposition of $\mathrm{N}$ levels there is a redundancy of $\mathrm{N}$ in the wavelet coefficients. Translation invariance property of DWT is overcome in SWT.

In this work, we are proposing an image resolution enhancement technique which removes drawbacks of interpolation \& DWT \& generates sharper high resolution image. The proposed technique uses DWT to decompose a low resolution image into different sub-bands, LL, LH, HL \& HH. Then the three high frequency sub-band images( $\mathrm{LH}, \mathrm{HL} \& \mathrm{HH}$ ) have been interpolated by enlargement factor of 2 using bi-cubic interpolation. The high frequency sub-bands obtained by SWT of the input image are being incremented into the interpolated high frequency sub-bands of DWT.. Also, the input image is interpolated separately. Finally, corrected interpolated high frequency sub-bands and interpolated input image are combined by using inverse DWT (IDWT) to achieve a high resolution output image. The proposed technique is compared with conventional and state-of-art image resolution enhancement techniques. The conventional techniques used are the following:

1)Interpolation techniques:

a) Nearest neighbor,

b) Bilinear interpolation and

c) Bi-cubic interpolation[2]

2) New Edge Directed Interpolation[6]

3) Wavelet Zero padding[8]

4) Complex wavelet transform[3]

5) Discrete wavelet Transform[11]

6) Regularity Preserving image interpolation.

If we compare the results of all these methods \& proposed technique the results are better. If we compare visual \& qualitative results of proposed technique with previous techniques then proposed technique gives better results than these all techniques.

\section{Proposed Method:}

To overcome the edge \& high frequency losses in above techniques, we propose a new resolution enhancement technique which combines DWT , SWT \& interpolation.

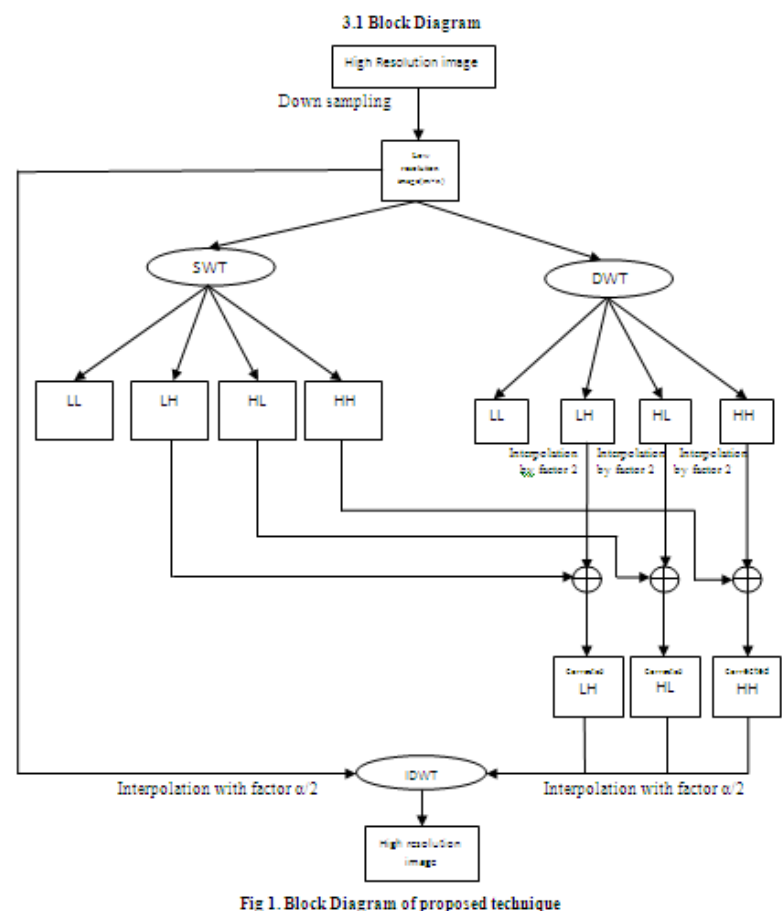


The fig1 shows block diagram of the proposed technique. The main blocks present in these method are

i) Original high Resolution image

ii) Down-sampled Low Resolution image.(Down-sampled by using 2 times dwt using Haar transform)

iii) Discrete wavelet transform

iv) Stationary wavelet transform

v) Interpolation of added sub-bands \& input image

vi) Inverse DWT.

vii) High resolution image

\subsection{Methodology:}

In the proposed technique first high resolution image is taken. Low resolution image is obtained by Down-sampling original high resolution image using 2 times $2 \mathrm{D}$ dwt using Haar transform .In this correspondence, one level 2D DWT (with Haar as wavelet function) is used to decompose an input image into different sub-band images.[10] Three high frequency sub-bands (LH, HL, and HH)contain the high frequency components of the input image. In the proposed technique, bi-cubic interpolation with enlargement factor of 2 is applied to high frequency sub-band images. Down sampling in each of the DWT sub-bands causes information loss in the respective sub-bands. So SWT is employed to minimize this loss.

The interpolated high frequency sub-bands of DWT and the SWT high frequency sub-bands have the same size which means they can be added with each other. These bands are added. These new corrected high frequency sub-bands are interpolated further for higher enlargement by factor of $\boldsymbol{\alpha} / \mathbf{2}$. Also we know that in the wavelet domain, the low resolution image is obtained by low pass filtering of the high resolution image. Means low resolution i/p image is low frequency component of high resolution o/p image. Therefore, instead of using low frequency sub-band, which contains less information than the original high resolution image, Hassan Demirel and G. Anbarjafari [12] using the input image for the interpolation of low frequency sub-band image. Using input image instead of low frequency sub-band increases the quality of the super resolved image. Fig1 illustrates the block diagram.

By interpolating input image by $\alpha / 2$ and corrected high frequency sub-bands by $\alpha / 2$, and then by applying IDWT, as illustrated in Fig.1, the output image will contain sharper edges than the interpolated image obtained by interpolation of the input image directly. The $\mathrm{o} / \mathrm{p}$ image is enhanced by factor of $\alpha$. This is due to the fact that he interpolation of isolated high frequency components in high frequency sub-bands and using the corrections obtained by adding high frequency sub-bands of SWT of the input image. It will preserve more high frequency components after the interpolation than interpolating input image directly. Super resolved images are characterized by the high frequency content of those images. High frequency components signify the edges and minute details spread all over the image. We have used components of DWT and SWT to preserve high frequency components.

\subsection{Algorithm for DWT using Haar Transform.}

In this paper algorithm for single level 2D DWT is developed using Haar transform. In DWT decomposition the decomposition is done row by row and then column by column. For instance, here is the procedure for an $\mathrm{N} x \mathrm{M}$ image. Then filter each row and down-sample to obtain two $\mathrm{N} x(\mathrm{M} / 2)$ images, they are $\mathrm{L}$ and $\mathrm{H}$ images. The formulae used to do find $\mathrm{H}$ and $\mathrm{L}$ is given below. In traditional methods Daubechies 9/7 filters are used.[12].Instead of that here new algorithm is proposed using Haar transform which gives superiority in visual \& calculative results.(Peak Signal to Noise Ratio)

$$
\begin{gathered}
\mathrm{H}=\text { odd-even }=\mathrm{F} 1 ; \\
\mathrm{L}=\text { even }+ \text { round }(\mathrm{F} 1 / 2) ;
\end{gathered}
$$

Then filter each column and subsample the filter output to obtain four (N/2) x (M/2) images, by using the Eq.(2)

$$
\begin{gathered}
\mathrm{LH}=\text { odd }- \text { even }=\mathrm{F} 2 ; \\
\mathrm{LL}=\text { even }+ \text { round }(\mathrm{F} 2 / 2) ; \\
\mathrm{HH}=\text { odd }- \text { even }=\mathrm{F} 3 ; \\
\mathrm{HL}=\text { even }+ \text { round }(\mathrm{F} 3 / 2) ;
\end{gathered}
$$




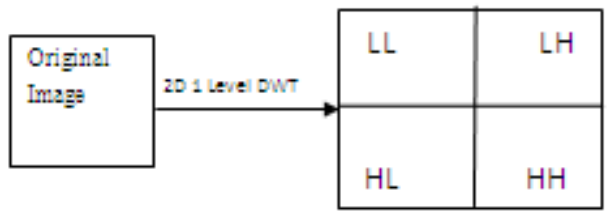

Fig 2. Decomposition using 2D 1 Level DWT

\subsection{Stationary Wavelet Transform (SWT).}

The Discrete Wavelet Transform is not a time- invariant transform. The way to restore the translation invariance is to average some slightly different DWT, called decimated DWT, to define the stationary wavelet transform (SWT).The Stationary wavelet transform (SWT) is a wavelet transform algorithm designed to overcome the lack of translation-invariance of the discrete wavelet transform (DWT). Translation-invariance is achieved by removing the down samplers and up samplers in the DWT and up sampling the filter coefficients. The SWT is an inherently redundant scheme as the output of each level of SWT contains the same number of samples as the input, so for a decomposition of $\mathrm{N}$ levels there is a redundancy of $\mathrm{N}$ in the wavelet coefficients.

\section{Results \& Discussions.}

Proposed method has been tested with standard set of test images (Lena, Elaine, baboon \& pepper,). The high resolution standard bench mark input images(size $512 \times 512)$ are taken. The high resolution $\mathrm{i} / \mathrm{p}$ images are down-sampled to $128 \times 128 \&$ low resolution image is obtained. This low resolution image is enhanced to $512 \times 512$ by proposed method. The quantitative (Peak signal to noise ratio) and visual results are superior over the conventional image resolution enhancement techniques. The original high resolution image is used as ground truth image to calculate Peak signal to noise ratio. The algorithm is applied on different images. But due to lack of space few are shown in Fig.3 The algorithm using Haar transform[10] is used for down sampling of image from $512 \times 512$ to $128 \times 128$

The performance is calculated by calculating Peak Signal to Noise ratio (PSNR) as given in equation (3).PSNR is performance measure for image quality.

$P S N R=10 \log _{10}\left[\frac{255 \times 255}{\mathrm{MSE}}\right]$

MSE - Mean Square error is calculated as

MSE $=\sum_{i=1}^{k} \sum_{j=1}^{y} \frac{\left(\mid A_{i j}-B_{i j} l\right)^{2}}{x}$

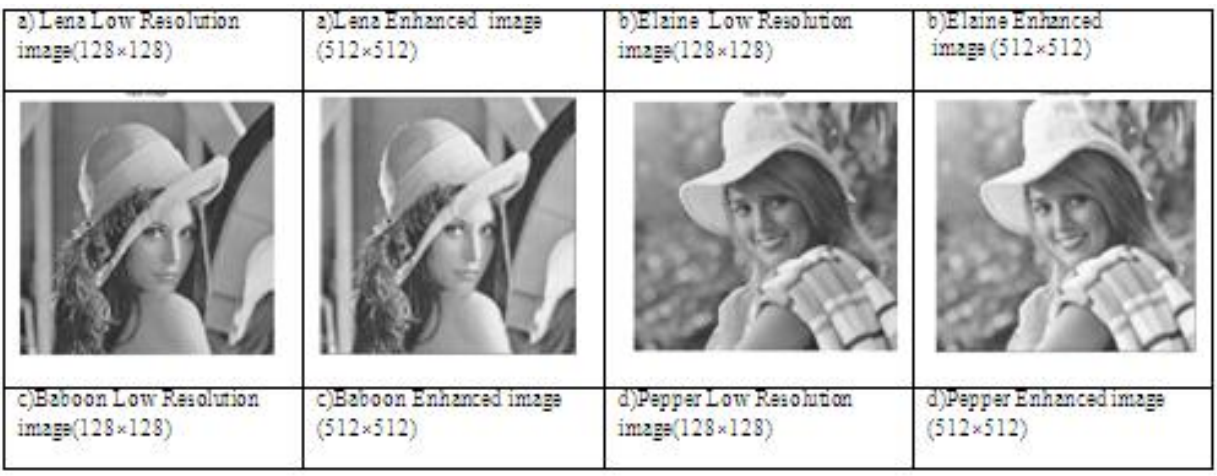

Fig.3 shows the results obtained with images of Lena, Elaine, Baboon pepper, Low resolution image along with high resolution images obtained by proposed technique is shown. Table-I shows that the proposed method works better than any prior methods. Edges and other minute details are more enhanced in 


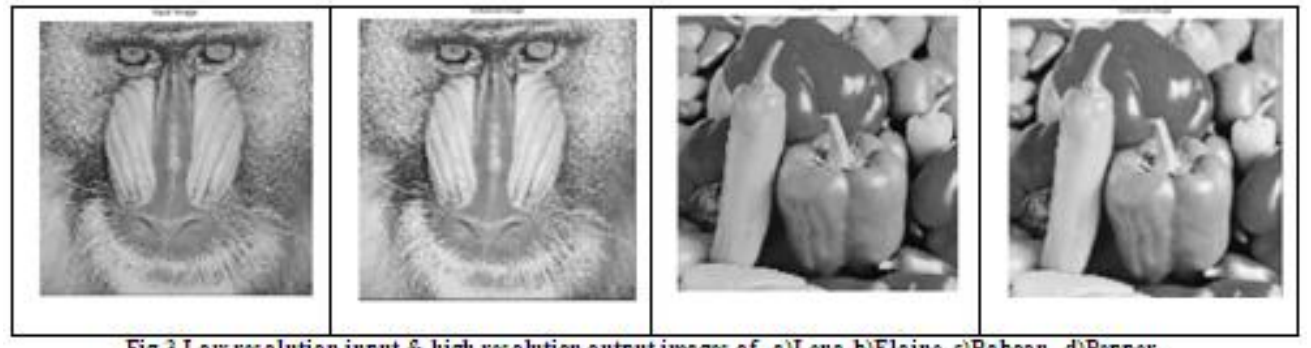

Fig. 3 Low resolution input \& high res olution output images of a)Lena b)Elaine c)Baboon d)Pepper

Table (1):- PSNR values (DB) for images enhanced from $128 \times 128$ to $512 \times 512(\alpha=4)$

\begin{tabular}{|c|c|c|c|c|}
\hline \multicolumn{6}{|c|}{ PSNR values of images in fig.(3). Images enhanced from $128 \times 128$ to $512 \times 512(\alpha=4)$} \\
\hline Method & Lena & Elaine & Baboon & Pepper \\
\hline Bilinear & 26.34 & 25.38 & 20.51 & 25.16 \\
\hline Bi-cubic[2] & 26.86 & 28.93 & 20.61 & 25.66 \\
\hline WZP(db9/7) & 28.84 & 30.44 & 21.47 & 29.57 \\
\hline DWT[11] & 34.79 & 32.73 & 23.29 & 32.19 \\
\hline CWT[3] & 33.74 & 33.05 & 23.12 & 33.01 \\
\hline SWT\&DWT(db9/7)[12] & 34.82 & 35.01 & 23.87 & 33.06 \\
\hline Proposed method & 35.53 & 36.65 & 34.42 & 35.53 \\
\hline
\end{tabular}

proposed method. Close observation of figure. 3 reveals that in proposed method high frequency content of image are better preserved.

\section{Conclusion}

From above TABLE $1 \&$ visual results in fig. 3, it is clear that the application of this method is successful for resolution enhancement. PSNR of the images Lena, Elaine, baboon pepper by using conventional methods ( bi-linear, bi-cubic, nearest neighbor interpolation, DWT ) and proposed method are listed in table I. Table I shows proposed method's superiority over conventional methods .

We have proposed new algorithm for decomposition of DWT. Instead of using db9/7 filter for DWT2 the LL, LH, HL \&HH are calculated by using Eq.(1) \&Eq.(2).We corrected the HF components of DWT according to SWT components. So edge preservation is better \& there is no loss of high frequency components. We have also provided subjective and objective comparison of resultant images. We have compared proposed method with state of the art methods. PSNR table shows the superiority of the proposed method over conventional methods.

\section{References}

[1]. H. Demirel, G. Anbarjafari, and S. Izadpanahi, "Improved motion-based localized super resolution technique using discrete wavelet transform for low resolution video enhancement," in Proc. 17th Eur. Signal Process. Conf., Glasgow, Scotland, Aug. 2009, pp. 1097-1101.

[2]. Robert G. Keys," cubic convolution interpolation for digital image processing”, IEEE transactions on acoustics, speech and signal processing, vol-29, no.6, December 1981

[3]. H.Demirel and G. Anbarjafari, "Satellite image resolution enhancement using complex wavelet transform," IEEE Geo- science and Remote Sensing Letter, vol. 7, no. 1, pp. 123-126, Jan. 2010.

[4]. C. B. Atkins, C. A. Bouman, and J. P. Allebach, "Optimal image scaling using pixel classification," in Proc. Int. Conf. Image Process Oct. 7-10, 2001, vol. 3, pp. 864-867.

[5]. W. K. Carey, D. B. Chuang, and S. S. Hemami, "Regularity-preserving image interpolation," IEEE Trans. Image Process. , vol. 8, no. 9, pp.1295-1297, Sep. 1999

[6]. X. Li and M T. Orchard, "New edge-directed interpolation,” IEEE Trans. Image Process. , vol. 10, , pp. 1521-1527, Oct. 2001.

[7]. K. Kinebuchi, D. D. Muresan, and R. G. Baraniuk, "Wavelet-based statistical signal processing using hidden Markov models," in Proc. Int. Conf. Acoustics., Speech Signal Process., 2001, vol.3, pp. 7-11.

[8]. S. Zhao, H. Han, and S. Peng, "Wavelet domain HMT-based image super resolution," in Proc. IEEE Int. Conf. Image Process. , Sep. 2003,vol. 2, pp. 933-936.

[9]. A. Temizel and T. Vlachos, "Wavelet domain image resolution enhancement using cycle-spinning," Electron. Letter ., vol. 41, no. 3, pp 119-121, Feb. 3, 2005.

[10]. P. Phanindra, J. Chinna Babu, V. Usha Shree, VLSI Implementation of Medical Image Fusion Using Haar Transform , International Journal of Scientific \& Enginee ring Research, Volume 4, Issue 9, September -2013,ISSN 2229 -5518

[11]. G. Anbarjafari and H. Demirel, "Image super resolution based on interpolation of wavelet domain high frequency sub-bands and the spatial domain input image,” ETRI J., vol. 32, no. 3, pp. 390-394,Jun. 2010.

[12]. Demirel, H. and G. Anbarjafari, IMAGE Resolution Enhancement by Using Discrete and Stationary Wavelet Decomposition. Image Processing, IEEE Transactions on, 2011.20(5).

[13]. Y. Piao, I. Shin, and H. W. Park, "Image resolution enhancement using inter-sub-band correlation in wavelet domain," in Proc. Int. Conf. Image Process., 2007, vol. 1, pp. I-445-448. 